Original Research Paper

\title{
Correlating and Modeling of Extracted Features from PVT Images of Composites using Optical Flow Technique and Weight Elimination Algorithm Optimization [OFT-WEA]
}

\author{
Mahmoud Zaki Iskandarani \\ Al-Ahliyya Amman University, \\ Faculty of Engineering, Department of Electronics and Communications Engineering, Jordan
}

\author{
Article history \\ Received: 20-02-2017 \\ Revised: 28-08-2017 \\ Accepted: 13-09-2017 \\ Email: m.iskandarani@hotmail.com
}

\begin{abstract}
A new approach to the use and implementation of Optical Flow technique is presented. The technique extracts features from presented images as a function of reference image and produces percentage of matching between the reference and tested images. The new approach in using Optical Flow lies in replacing the motion part of the algorithm with differential time related changes in an infrared thermal image sequence with frames of images taken as a result of applying the Pulse Video Thermography (PVT) technique. The sequence of images or frames is obtained for the tested structures of composites before and after impact damage. The resulted data of the tested images is used to establish mathematical model that can be used to predict impact energy from collected features or predict expected features from knowing impact damage level. To optimize the mathematical model, a new way of using Neural Networks is employed, which aims at obtaining a best fit for the used variables in the mathematical model, hence resulting in a better testing interpretation and more accurate prediction and classification of image features to improve future composite structures designs. The Neural Network Weigh Elimination Algorithm (WEA) is used and proved effective in predicting areas of damage.
\end{abstract}

Keywords: Composites, Optical Flow, Feature Extraction, Correlation, Impact Damage, Image Processing, Neural Networks, Modeling, PVT

\section{Introduction}

Composite materials are a function of their internal structures, which is optimized to obtain desired mechanical properties. The fibres on their own are generally of little practical use but with a welldesigned combination of fibres and matrix a reliable component with good performance is produced and vitally used in demanding environments such as vehicles, airplanes and space craft industries.

Integrity of a material is based on quality of fabrication as the designed specifications are fulfilled. Hence, it is important to be able to control the manufacturing and to inspect the materials for their structural arrangements of fibres, matrix and defects which most often have a detrimental effect on the composite properties and contributes to the readiness of a manufactured structure to easily be damaged.

For practical and economical use of composites, it is very important to know not only the level of damage in the material but also its effect on the properties and functionality of the material, so that enough evidence will be available for accepting or rejecting a component.
Testing a composite structure involves:

- During manufacturing inspection

- During service inspection

The object of both tests is to determine an existing damage to the structure and its effect on the component performance and functionality, thus specify its limits.

Two main areas of importance when testing structures:

- Damage detection and identification

- Analysis and monitoring of damage progression

Thus, in order to develop appropriate inspection technology for fiber-reinforced composite materials, it is necessary that the development of detection capabilities be coupled with development of the technology that is capable of assessing the influence of damage upon strength and stiffness.

Optical Flow is a known method of estimating field deviation between two similar images. It measures the 
spatial and temporal variations. It looks for changes over time and for surface and composition changes over a period of time, where two images or more are taken.

Neural networks play a critical role as data analysis and optimization tool, developed to speed the damage detection process. The Optical Flow feature extraction method utilizes a reference sample with Weight Elimination Algorithm operating on a designed Neural Network structure. The new approach is used for pattern recognition and impact energy correlation to extracted features in a PVT image (Usmantiagar et al., 2013; Katunin et al., 2014; Ma and Liu, 2016; Wang et al., 2016; Liang et al., 2016; Yang et al., 2016; Balcıoğlu et al., 2016; Hessamodin et al., 2016; Rajendran and Srinivasan, 2015).

\section{Background}

Composite structural testing helps to ensure that materials enter service in a functional condition. Too little sensitivity in the testing process would allow failure to occur and too much sensitivity would waste perfectly good structures.

The basic contributions to the effectiveness and reliability of testing comes from the nature of the structure itself, the environment in which the structure is expected to operate and the kinds of defects expected to occur. From this information it is possible to estimate the damage type and level, which might lead to failure.

Having estimated the nature of damage, which could lead to failure, suitable techniques is developed and based on understanding of the way in which damage mechanism occurs and the best way for its detection. The chosen technique needs to be calibrated using reference structures. This includes undamaged samples and artificially damaged ones.

The developed technique needs to be able to detect damage at three major stages:

- Material processing

- Through manufacturing

- In service

This is essential since damage have the tendency to occur over time and under load applications.

Testing can be employed to detect inclusions and flaws that differ in their heat transfer properties from the undamaged structure. When external heat is applied, the presence of damage affects the normal heat flow pattern of the structure. If this heat propagation is altered sufficiently, a temperature distribution profile can be realized.

Thermal Imaging (Electronic Thermography) is essentially an infrared radiation from the sample which subsequently converted into an electrical signal generating a real-time thermal image.

As a result of damage, the thermal characteristics of the materials evidently affect the resulting image which has dynamic characteristics depending on the thermal diffusivity of the material.

To provide sufficient temperature contrast, the material requires a sufficient quantity of heat. The actual temperature rise of a structure is affected by:

- The level of energy applied to the structure under test

- The rate of absorption

- The dynamic thermal properties of the material

Since the steepness of the temperature gradient provides the required "contrast" between damaged and undamaged areas, a fast pulse as a source of heat must be used for a rapid temperature rise.

Temperature difference is greatest shortly after the initial absorption of thermal energy and decreases over time. Therefore, it is necessary to measure temperature over an adequate time period.

The previous highlights the need for an intelligent classification system, which is flexible enough to accommodate different boundary conditions with complex non-linear behavior with capabilities of generalization and prediction.

Quality monitoring of structures requires automated test and assessment technique, which can be further enhanced using an Intelligent Assessment (IA), where an automated inspection system is used to assess the quality of images obtained for structures in relation to reference images and also predict future structural behavior under different conditions.

Neural Networks can directly map the characteristics of a material. Work has been carried out to build and apply Neural Networks models to characterize, map and predict parameters such as, temperature, effective strain, strain rate, creep, among others.

Optical Flow is a popular method of estimating the difference between two images due to a time related function that correlate between two successive images. It measures intensity changes. In general, there are three common assumptions to approximate Optical Flow values:

- Brightness constancy

- Spatial coherence

- Temporal persistence

Optical Flow can be represented as:

$\nabla I \bullet \vec{f}+I_{t}=0$

Where:

$I(x, y, t)$ is the image intensity at time $\mathrm{t}$ located at spatial point $(x, y) . \nabla \mathrm{I}=\left(I_{x}, I_{y}\right)$ is the spatial gradients and $I_{t}$ is the temporal gradient of the intensity function. If 
point of interest in the image is initially positioned at $(x, y)$ and it moves through a distance $(d x, d y)$ after the change in time of $d t$. The flow vector $\vec{f}$ will consist horizontal and vertical components, $\vec{f}=\left[p=\frac{d x}{d t}, q=\frac{d y}{d t}\right]$ which denotes the horizontal and vertical components of the optical flow. Such point of interest and its movement can represent different types of damage propagating through a structure over time. In addition using object subtraction as part of the Optical Flow algorithm can represent the presence of damage and more specifically, impact damage, fiber extraction, cracks, foreign bodies in the structure, chemical interaction with the structure.

Weight Elimination Algorithm is used to carry out weight decay process. It minimizes a modified error function which is formed by adding an extra term related to training error to the original error function.

The added error term controls the dynamics of large weights, as it causes weights under consideration to converge with a much smaller values. Large weights can adversely affect network generalization depending on their position in the network. If the weights with large values are between input layer and hidden layer, they can result in the output function to be too rough. However, if they lie between the hidden layer and the output layer, they can result in outputs beyond the range of the data. Hence, large weights can cause excessive variance of the output, which cause the neural structure to be unstable with values outside the range of the output activation function (Peng et al., 2015; Salazar et al., 2012; Qingsong et al., 2014; Duchi et al., 2011; May et al., 2013; Bahadorinia et al., 2014; Kumar and Harikumar, 2015; Dai et al., 2015; Barry et al., 2016; Ashwini and Yuvaraju, 2016).

Weight Elimination mathematical model describes the dynamic changes in Neural Network convergence in relation to error functions. The overall weight elimination error function is presented by Equation (2) and it consists of two parts represented in the overall expression in Equation (2):

- Original error function described by Equation (3)

- Training error function described by Equation (4)

$E_{\text {overall }}=E_{\text {original }}+E_{\text {training }}$

$E_{\text {original }}=\frac{1}{2} \sum_{k}\left(d_{k}-o_{k}\right)^{2}$

$E_{\text {training }}=\beta\left(\sum_{j k} \frac{\left(\frac{w_{j k}}{w_{n e t}}\right)^{2}}{1+\left(\frac{w_{j k}}{w_{n e t}}\right)^{2}}\right)$
Where:

$E_{\text {overall }}$ : The combined overhead function that includes the initial overhead function, $E_{\text {original }}$ and the weight-elimination term $E_{\text {training }}$

$\beta$ : Weight-Reduction factor

$w_{j k}$ : Represents the individual weights of the neural network model

$w_{\text {net }}: \quad$ Scale parameter

$d_{k}$ : $\quad$ Desired output

$O_{k}$ : Actual output

The dynamic weight changes is calculated using Equation (5):

$\Delta w_{j k}=\left(-\eta \frac{\partial E_{\text {original }}}{\partial w_{j k}}\right)-\left(\beta \frac{\partial E_{\text {training }}}{\partial w_{j k}}\right)$

Where:

\section{$\eta$ : Learning rate}

The parameter, $w_{\text {net }}$, is a scale parameter and chosen to be the smallest weight from the last epoch or set of epochs to force small weights to zero $w_{\text {net }}$ guides the computing algorithm to find solutions with either fewer large weights or many small weights, depending on the $w_{\text {net }}$ values small or large.

This work introduces a new method that maps motion used in the optical flow technique and algorithm to structural change in composites caused by impact damage. The mentioned two dimensional change which affects intensity due to motion can be mapped onto two dimensional changes in intensity due to impact damage in composites as a function of time. The developed mathematical model is further optimized by neural networks Algorithm (WEA) which is used in a new way to fine tune parameters in the model (Baghaie et al., 2017; Solari et al., 2015).

\section{Materials and Methods}

RIM composite components are shaped into rectangles $(130 \times 150 \mathrm{~mm})$. Drop weight system is used to induce impact damage at various energy levels using $1.35 \mathrm{Kg}$ weight.

Images obtained for all tested components pre and post impact using PVT technique. Reference (prior to impact) sample images are used to cross correlate with the impacted samples, then optical flow technique is used and features extracted. The resulting feature maps for tested samples are used to derive mathematical expression governing the behavior of the Optical Flow technique in relation to extracted features and impact energies, then the resulting maps encoded into arrays of data to correlate Thermal Flow features with optical flow features. The reference encoding feature map is shown in Fig. 1. WEA is used to further improve the behavior of the obtained model. 


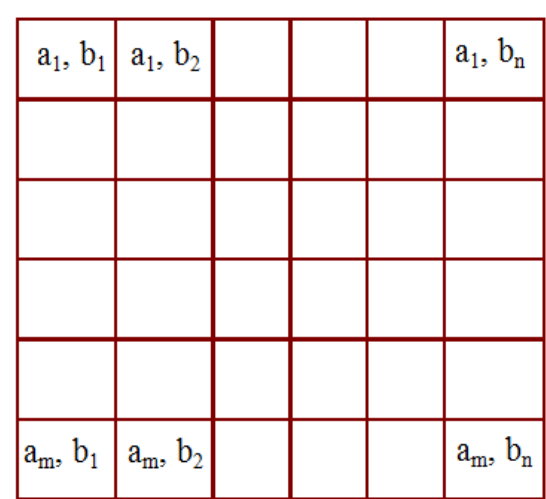

Fig. 1. Encoding reference map

For Pulse Video Thermography (PVT) the equipment used consisted of heat source and thermal imaging system. The heat source is adequately fast pulse, in order that there is a rapid temperature rise, as the steepness of the temperature gradient provided the differential required between damaged and undamaged areas. Pulse obtained by discharging several Kilojoules of energy through each of two Xenon flash tubes, which are directed at the component under test. The thermal imaging part of the experiment was carried out using an advanced thermal camera. Each thermal event was recorded directly on a specific storage.

\section{Results}

Figure 2 to 5 show the Optical Flow feature maps Images for the tested RIM composites, while Table 1 show tolerances effect and Table 2 show the results of composite testing and feature extraction.

\section{Analysis and Discussion}

The process of analyzing and modeling the relationship between impact energy and extracted features is based on three elements:

- Feature extraction tolerance

- Impact energy level

- Impact load incident angle

Table 1 shows accuracy values for extracted features as a function of applied tolerance values. From the Table, it is clear that as tolerance values increase, extracted features accuracy decreases. This leaves only one usable value of tolerance, which is 0.05 that can be used safely for such optical flow images. Tolerance reduces the computing time and speeds up calculations, which is a favorable feature for real time testing, at the same time and for critical tests, the use of wide tolerance values is undesirable.
Figure 6 shows a plot for the values in Table 2. The plot shows the power curve or exponential rise and decay curves. Within the curve are the real testing values with Table 3 showing the obtained values from the plot in Fig. 6, it is evident that as the impact energy increases, matched features decreases and a marked difference arises between the reference image and the tested image.

From both Fig. 6 and Table 3, Equation are obtained covering both matched and mismatched features extracted as a function of impact energy presented in (6) and (7):

Matched $=F\left[e^{-\left(\frac{I E}{0.63^{*} F}\right)}\right]$

Mismatched $=F\left[1-e^{-\left(\frac{I E}{0.63^{*} F}\right)}\right]$

Where:

$\mathrm{IE}=$ Impact Energy

$\mathrm{F}=$ Extracted Features

From Equation (6) and (7), Table 4 is obtained with Fig. 7 illustrate the simulated and approximated relationship between impact energy and extracted features. It is noticed that there is a difference in the produced values and that is due to the approximation effect, but still within acceptable limits.

The approximation obtained through Equation (6) and (7) can further be optimized using the neural networks shown in Fig. 8. Table 5 presents values obtained using the designed neural structure.

From Table (3) and (4), the two originally found values for the match and mismatch curves at which half the features are matched and the other half are mismatched are $15.75 \mathrm{~J}$ and $17.5 \mathrm{~J}$. When used in the neural engine, the Engine predicted a value in between the two values, which is $16.15 \mathrm{~J}$. Hence, using neural networks with Weight Elimination Algorithm (WEA), both the original obtained values from the test data in Fig. 6 and the mathematically obtained plot in Fig. 7 are optimized, producing a stable and more accurate prediction curve, which also conforms to the mathematical expressions in Equation (6) and (7), as shown in Fig. 9.

From experiments and for all practical purposes, not all impacts occur at normal to the surface; hence Equation (8) and (9) describe the effect of impact at an incident angle $\phi$. Both equations cover one aspect of incident angle effect and that is the normal to the surface part of the vector, which is suspected to cause not only internal damage, but also surface damage:

$\operatorname{Matched}\left(\varphi_{\text {incident angle }}\right)=F\left[e^{-\left(\frac{I E \sin \varphi}{0.63^{*} F}\right)}\right]$ 
Mahmoud Zaki Iskandarani / Journal of Computer Sciences 2017, 13 (9): 371.379 DOI: $10.3844 /$ jcssp.2017.371.379

Table 1. Tolerance effect on feature extraction

\begin{tabular}{|c|c|c|c|c|}
\hline \multirow[b]{2}{*}{ Tolerance } & \multicolumn{4}{|c|}{ Extraction accuracy } \\
\hline & Ref & S2 & S3 & S4 \\
\hline 0.00 & 100 & 100 & 100 & 100 \\
\hline 0.05 & 100 & 100 & 100 & 100 \\
\hline 0.10 & 100 & 70 & 65 & 61 \\
\hline 0.15 & 100 & 57 & 52 & 57 \\
\hline 0.20 & 100 & 53 & 44 & 52 \\
\hline 0.25 & 100 & 50 & 39 & 48 \\
\hline 0.30 & 100 & 47 & 35 & 44 \\
\hline 0.35 & 100 & 47 & 35 & 30 \\
\hline 0.40 & 100 & 40 & 30 & 26 \\
\hline 0.45 & 100 & 33 & 26 & 26 \\
\hline 0.50 & 100 & 27 & 26 & 17 \\
\hline
\end{tabular}

Table 2. Feature extraction as a function of impact energy

\begin{tabular}{llllll}
\hline Sample & Impact energy $(\mathrm{J})$ & No of tests & Features & Matched & Mismatched \\
\hline Ref & 0 & 1000 & 1008 & 1008 & 0 \\
S2 & 28.6 & 1000 & 1008 & 215 & 793 \\
S3 & 42 & 1000 & 1008 & 155 & 853 \\
S4 & 55.6 & 1000 & 1008 & 139 & 869 \\
\hline
\end{tabular}

Table 3. Impact energy and optical feature values obtained from figure 6 including real testing data

\begin{tabular}{llll}
\hline Impact energy $(\mathrm{J})$ & Features & Matched & Mismatched \\
\hline 0.00 & 1008 & 1008 & 0 \\
5.00 & 1008 & 860 & 148 \\
10.0 & 1008 & 718 & 290 \\
15.0 & 1008 & 568 & 440 \\
17.5 & 1008 & 504 & 504 \\
20.0 & 1008 & 428 & 580 \\
25.0 & 1008 & 300 & 708 \\
28.6 & 1008 & 215 & 793 \\
30.0 & 1008 & 190 & 818 \\
35.0 & 1008 & 150 & 858 \\
40.0 & 1008 & 155 & 853 \\
42.0 & 1008 & 155 & 853 \\
45.0 & 1008 & 150 & 858 \\
50.0 & 1008 & 140 & 868 \\
55.6 & 1008 & 139 & 869
\end{tabular}

Table 4. Impact energy and optical feature values obtained from Equation (6) and (7)

\begin{tabular}{|c|c|c|c|}
\hline Impact energy $(\mathrm{J})$ & Features & Matched & Mismatched \\
\hline 0 & 1008 & 1008 & 0 \\
\hline 5 & 1008 & 809 & 199 \\
\hline 10 & 1008 & 649 & 359 \\
\hline 15 & 1008 & 516 & 492 \\
\hline 15.75 & 1008 & 504 & 504 \\
\hline 20 & 1008 & 414 & 594 \\
\hline 25 & 1008 & 332 & 676 \\
\hline 28.6 & 1008 & 283 & 725 \\
\hline 30 & 1008 & 267 & 741 \\
\hline 35 & 1008 & 212 & 796 \\
\hline 40 & 1008 & 170 & 838 \\
\hline 42 & 1008 & 155 & 853 \\
\hline 45 & 1008 & 136 & 872 \\
\hline 50 & 1008 & 109 & 899 \\
\hline 55.6 & 1008 & 85 & 923 \\
\hline
\end{tabular}


Mahmoud Zaki Iskandarani / Journal of Computer Sciences 2017, 13 (9): 371.379 DOI: $10.3844 /$ jessp.2017.371.379

Table 5. Impact energy and optical feature values obtained using WEA

\begin{tabular}{|c|c|c|c|}
\hline Impact energy (J) & Features & Matched & Mismatched \\
\hline 0.000 & 1008 & 1008 & 0 \\
\hline 5.000 & 1008 & 966 & 42 \\
\hline 10.00 & 1008 & 817 & 191 \\
\hline 15.00 & 1008 & 560 & 448 \\
\hline 15.75 & 1008 & 523 & 485 \\
\hline 16.15 & 1008 & 504 & 504 \\
\hline 17.50 & 1008 & 446 & 562 \\
\hline 20.00 & 1008 & 360 & 648 \\
\hline 25.00 & 1008 & 256 & 752 \\
\hline 28.60 & 1008 & 215 & 793 \\
\hline 30.00 & 1008 & 204 & 804 \\
\hline 35.00 & 1008 & 176 & 832 \\
\hline 40.00 & 1008 & 159 & 849 \\
\hline 42.00 & 1008 & 155 & 853 \\
\hline 45.00 & 1008 & 150 & 858 \\
\hline 50.00 & 1008 & 143 & 865 \\
\hline 55.60 & 1008 & 139 & 869 \\
\hline
\end{tabular}

Table 6. Impact energy and feature values obtained from equations (8) and (9)

\begin{tabular}{lllrr}
\hline Impact energy $(\mathrm{J})$ & Features & $\phi$ & Matched & Mismatched \\
\hline 0.00 & 1008 & 0 & 1008 & 0 \\
28.6 & 1008 & 30 & 532 & 476 \\
28.6 & 1008 & 45 & 410 & 598 \\
28.6 & 1008 & 60 & 336 & 672 \\
28.6 & 1008 & 90 & 283 & 725 \\
42.0 & 1008 & 30 & 398 & 610 \\
42.0 & 1008 & 45 & 269 & 739 \\
42.0 & 1008 & 60 & 202 & 806 \\
42.0 & 1008 & 90 & 155 & 853 \\
55.6 & 1008 & 30 & 292 & 716 \\
55.6 & 1008 & 45 & 175 & 833 \\
55.6 & 1008 & 60 & 120 & 888 \\
55.6 & 1008 & 90 & 85 & 923 \\
\hline
\end{tabular}

Fig. 2. Optical Flow map of PVT image of reference composite sample

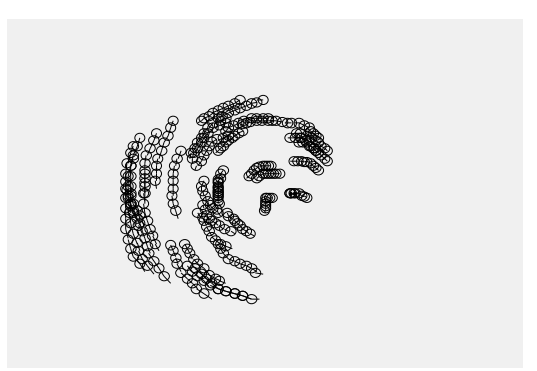

Fig. 3. Optical Flow map of $28.6 \mathrm{~J}$ impact damaged composite sample- S2

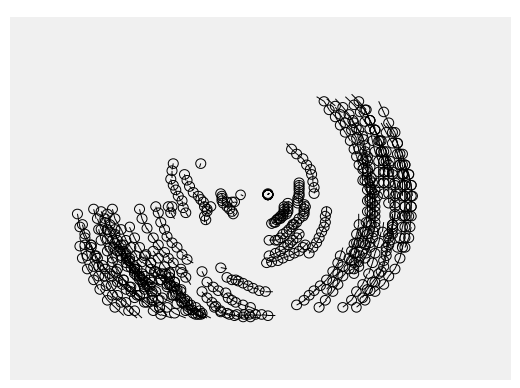

Fig. 4. Optical Flow map of 42J impact damaged composite sample-S3

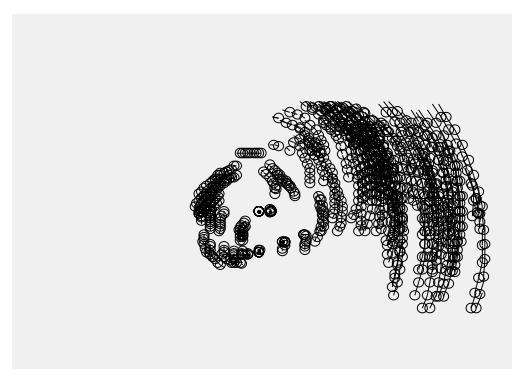

Fig. 5: Optical Flow map of 55.6J impact damaged composite sample -S4 

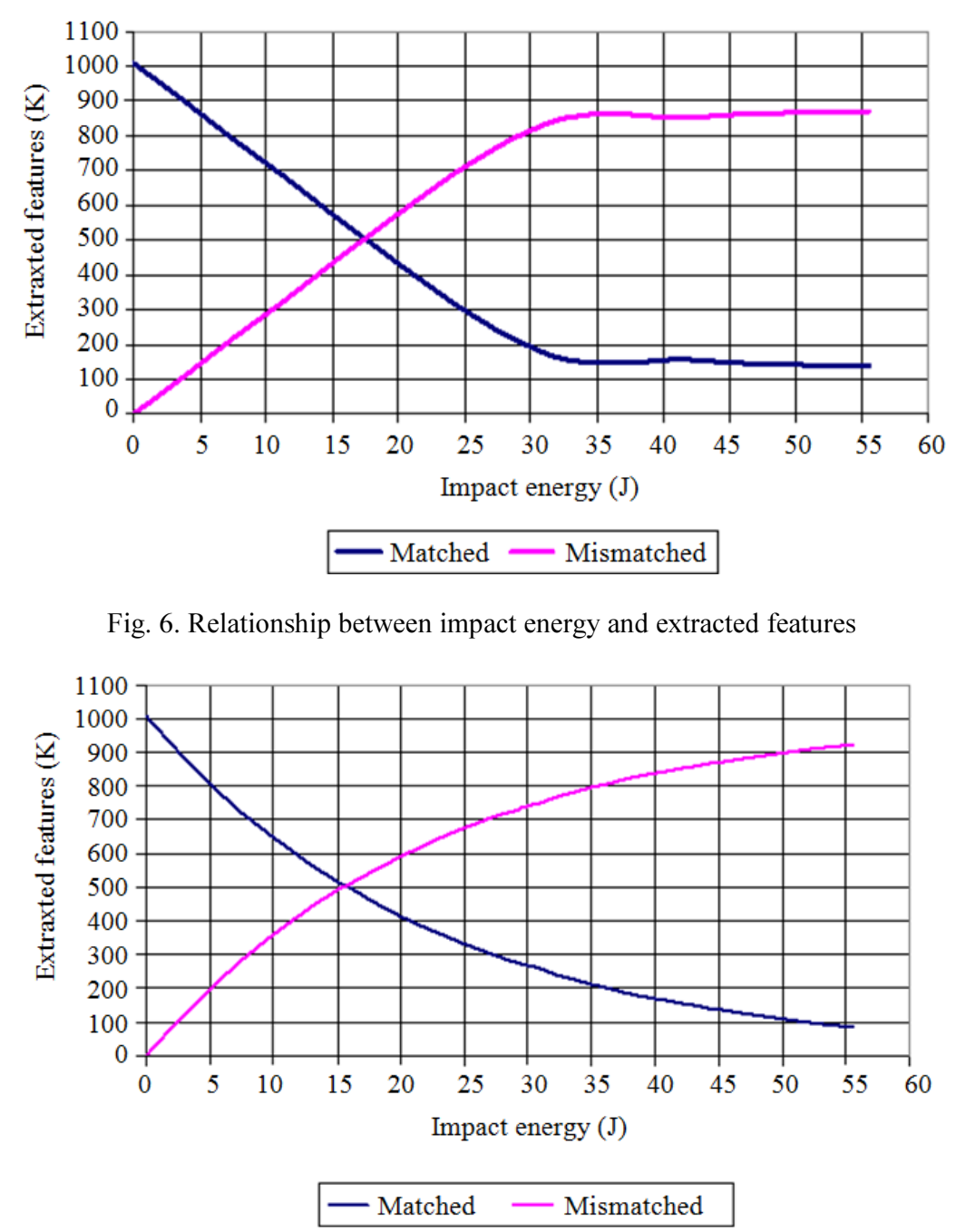

Fig. 7. Approximation for the relationship between impact energy and extracted features

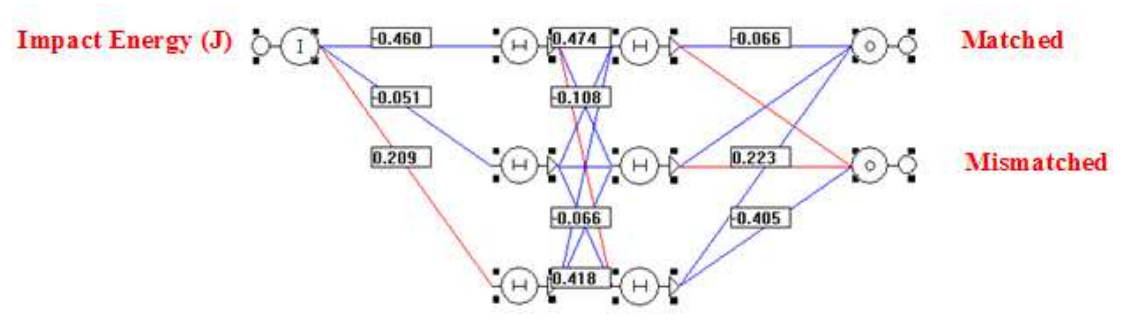

Fig. 8. Neural networks using Weight Elimination Algorithm (WEA) to predict matching and mismatching features

$$
\operatorname{Mismatched}\left(\varphi_{\text {incident angle }}\right)=F\left[1-e^{-\left(\frac{I E \sin \varphi}{0.63^{*} F}\right)}\right]
$$

Where:

$\phi=$ Angle to the normal of the surface

Results using Equation (8) and (9) are presented in Table 6.
Table 6 shows that as the incident angle increases and approaches the angle normal to the surface, the mismatched features increases, which is logical as the force of impact increases and for a certain expected and calculated impact energy as a function of impact load, weight and vertical distance, the actual impact energy will not have its desired effect and its actual value will be masked by the incident angle. 


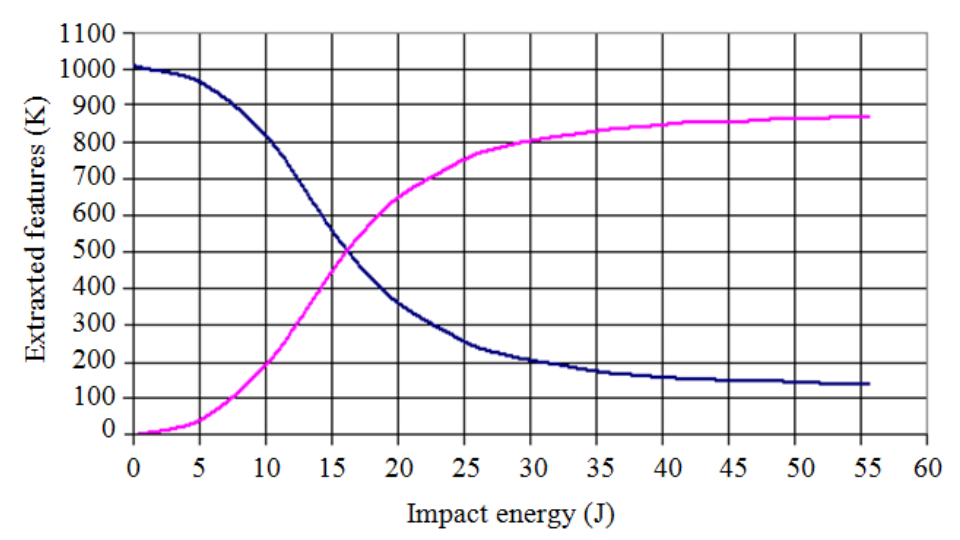

- Matched Mismatched

Fig. 9. Neural networks optimization describing the relationship between impact energy and extracted features

\section{Conclusion}

Combining PVT and energy flow in a mathematical model that enables approximate computations of Both Impact Energy and Extracted Features is a new way of dealing with damage detection in an testing environment. Effect of impact load incident angle is also an important issue, which is considered in the work. The approximation expressions are made more accurate using Neural Networks engine, which optimized and reproduced the best fit data to the type of composites tested.

\section{Ethics}

There are no ethical issues with this article.

\section{References}

Ashwini, B. and B. Yuvaraju, 2016. Feature extraction techniques for video processing in MATLAB. Int. J. Innovative Res. Comput. Commun. Eng., 4: 5292-5296. DOI: 10.15680/IJIRCCE.2016. 0404223

Baghaie, A., R. D'Souza and Z. Yu, 2017. Dense descriptors for optical flow estimation: A comparative study. J. Imaging, 3: 1-19.

Bahadorinia, A., A. Dolatabadi and A. Hajipour, 2014. A hybridized artificial neural network and optimization algorithms for the diagnosis of cardiac arrhythmias. Adv. Comput. Sci., Int. J., 3: 51-58.

Balcıŏlu, H., A. Seçkin and M. Aktaş, 2016. Failure load prediction of adhesively bonded pultruded composites using artificial neural network. J. Composite Mater., 50: 3267-3281. DOI: 10.1177/0021998315617998

Barry, T., M. Kisharaju, C. Nagarajah and S. Palanisamy, 2016. Defect characterisation in laminar composite structures using ultrasonic techniques and artificial neural networks. J. Composite Mater., 50: 861-871. DOI: $10.1177 / 0021998315584651$
Dai, H., H. Zhang and W. Wang, 2015. A multi wavelet neural network-based response surface method for structural reliability analysis. Comput. Aided Civil Infrastructure Eng., 30: 151-162. DOI: $10.1111 /$ mice. 12086

Duchi, J., E. Hazan and Y. Singer, 2011. Adaptive subgradient methods for online learning and stochastic optimization. J. Mach. Learn. Res., 12: 2121-2159.

Hessamodin, T., A. Milani, R. Seethaler and A. Heidarzadeh, 2016. On the Impact of manufacturing uncertainty in structural health monitoring of composite structures: A signal to noise weighted neural network process. Open J. Composite Mater., 6: 28-39. DOI: 10.4236/ojcm.2016.61004

Katunin, A., P. Przystałka, J. Guerediaga and A. Wronkowicz, 2014. Evaluation of impact damages in composites based on ultrasonic and optical images with optimized parameters. Mach. Dynamics Res., 38: 33-41.

Kumar, B. and R. Harikumar, 2015. Performance analysis of neural networks for classification of medical images with wavelets as a feature extractor. Int. J. Imaging Syst. Technol., 25: 30-40. DOI: $10.1002 /$ ima.22118

Liang, T., W. Ren, G. Tian, M. Elradi and Y. Gao, 2016. Low energy impact damage detection in CFRP using eddy current pulsed thermography. Composite Struc., 143: 352-361. DOI: $10.1016 /$ j.compstruct.2016.02.039

Ma, L. and D. Liu, 2016. Delamination and fiber-bridging damage analysis of angle-ply laminates subjected to transverse loading. Int. J. Imaging Syst. Technol., 50: 3063-3075. DOI: 10.1177/0021998315615647

May, P., E. Zhou and C.W. Lee, 2013. A comprehensive evaluation of weight growth and weight elimination methods using the tangent plane algorithm. Int. J. Adv. Comput. Sci. Applic., 4: 149-156. DOI: 10.14569/IJACSA.2013.040621 
Peng, F., J. Li and M. Long, 2015. Identification of natural images and computer-generated graphics based on statistical and textural features. J. Forensic Sci., 60: 435-443. DOI: 10.1111/1556-4029

Qingsong, Z., E. Jiaqiang, G. Jinke, L. Lijun and C. Tao et al., 2014. Functional link neural network prediction on composite regeneration time of diesel particulate filter for vehicle based on fuzzy adaptive variable weight algorithm. J. Inform. Computat. Sci., 11: 1741-1751. DOI: 10.12733/jics20103209

Rajendran, P. and S. Srinivasan, 2015. Identification of added mass in the composite plate structure based on wavelet packet transform. Strain, 52: 14-25. DOI: $10.1111 /$ str. 12154

Salazar, D.A, J. Ivan Velez and J.C. Salazar, 2012. Comparison between SVM and logistic regression: Which one is better to discriminate? Revista Colombiana De Estadistica, 35: 223-237.
Solari, F., M. Chessa, N.K. Medathati and P. Kornprobst, 2015. What can we expect from a V1-MT feed forward architecture for optical flow estimation? J. Image Commun., 39: 342-354.

Usmantiagar, R., P. Venegas, J. Guerediaga, L. Vega and I. Lopez, 2013. Feature extraction and analysis for automatic characterization of impact damage in carbon fiber composites using active thermography. NDT E. Int., 54: 123-132. DOI: 10.1016/j.ndteint.2012.12.011

Wang, F., J. Liu, Y. Liu and Y. Wan, 2016. Research on the fiber lay-up orientation detection of unidirectional CFRP laminates composite using thermal-wave radar imaging. Int. J. Imaging Syst. Technol., 84: 54-66. DOI: $10.1016 /$ j.ndteint.2016.08.002

Yang, R., Y. He and H. Zhang, 2016. Progress and trends in nondestructive testing and evaluation for wind turbine composite blade. Renewable Sustainable Energy Rev., 60: 1225-1250. DOI: $10.1016 /$ j.rser.2016.02.026 